\title{
Effect of rainfall on the reliability of an infinite slope
}

\author{
J. Yuan, I. Papaioannou, C. M. Mok \& D. Straub \\ Engineering Risk Analysis Group, \\ Technische Universität München, Munich, Germany
}

\begin{abstract}
Rainfall is one of the most common factors triggering landslides, since infiltration of water into the soil has a significant impact on pore water pressure buildup that affects slope stability. In this study, the influence of the wetting front development on the reliability of an infinite slope is analyzed. The failure condition of the slope is expressed in terms of the factor of safety. Rainfall infiltration is simulated by a timedependent model, based on the Green and Ampt assumptions. The vertical variability of the saturated hydraulic conductivity and soil strength parameters are modeled as random fields. The reliability of the slope is evaluated by Monte Carlo simulation. A numerical example demonstrates the influence of the vertical variability on the analysis results.
\end{abstract}

\section{INSTRUCTIONS}

Rainfall has a considerable influence on landslide occurrence (Polemio 1997, Corominas et al. 2002, Zêzere et al. 2008). In wet seasons, intense rainfall events often cause shallow slope failure. In such cases, the failure surface is approximately parallel to the ground surface and hence an infinite slope model can be used to represent the slope failure mechanism (Wu \& Abdel-Latif 2000, Santoso et al. 2011). Since surficial infiltration is predominately driven by gravitation, the infinite slope is often simplified in practice as a column subject to vertical infiltration, unless topography is very steep. It should be noted that some authors assumed that water percolates perpendicular to the sloping surface (Chen \& Young 2006, Muntohar \& Liao 2010)

In this paper, rainfall infiltration is modeled by applying the Green and Ampt assumptions for onedimensional vertical subsurface flow (Green \& Ampt 1911). Hence, it is assumed that the wetting front represents a clear border between the wetted and dry (in-situ condition prior to rainfall) soil zones. Further assuming that the water table is parallel to the ground surface, the infiltration model can be combined with the classical equation for infinite slope stability analysis to evaluate the timedependent behavior of the factor of safety of the slope.

Soil property values tend to vary in space, even within a lithologic layer that appears to be homogeneous (Phoon \& Kulhawy 1999). We model the inherent vertical variability of the strength parameters and saturated hydraulic conductivity as one-dimensional statistically homogeneous random fields. Through discretization of the random fields, the soil column is represented by a multilayered system whose layers have uniform parameters described by correlated random variables. Through Monte Carlo simulation, the statistics of the wetting front development and the slope factor of safety as well as the probability of failure of the slope are estimated. Moreover, the influence of the variability of the soil properties on the analysis results is investigated.

\section{TIME-DEPENDENT MODEL}

\subsection{Stability of infinite slope}

Consider a slice of an infinite slope, as shown in Figure 1. Assuming that the water table and potential slip surface are parallel to the ground surface, and according to the linear Mohr-Coulomb failure criterion, the factor of safety of the slip surface is defined as (Griffiths et al. 2011):

$F S=\frac{\left(\gamma H \cos ^{2} \beta-u\right) \tan \varphi^{\prime}+c^{\prime}}{\gamma H \sin \beta \cos \beta}$

in which, $\gamma$ is the average unit weight of the soil mass above the slip surface; $H$ is the depth of the slip surface; $\beta$ is the slope inclination; $u$ is the pore water pressure at the slip surface; $\varphi^{\prime}$ is the effective friction angle at the slip surface; $c^{\prime}$ is the effective cohesion at the slip surface. 


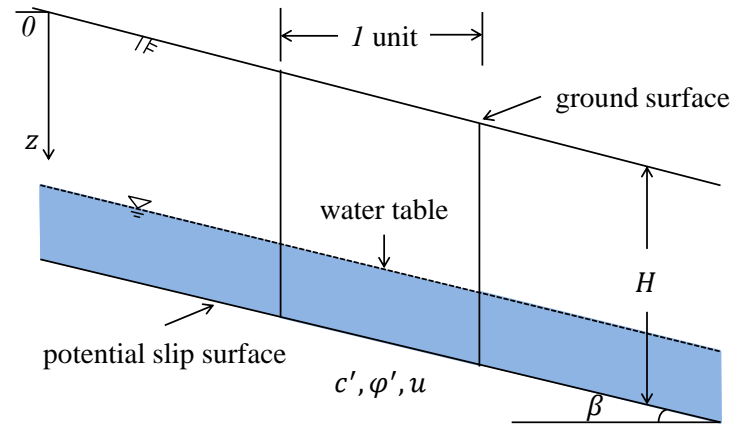

Figure 1. Typical unit slice from an infinite slope

\subsection{Stochastic model of soil parameters}

The stability of the slope depends on the shear strength of the soil at the slip surface, expressed in terms of the effective strength parameters $c^{\prime}$ and $\varphi^{\prime}$. Moreover, the infiltration process, which affects the pore water pressure $u$ during the wetting front development and the rise of the water table, is governed by the vertical saturated hydraulic conductivity $K$ of the soil. In this study, we account for the variability of the tangent of the friction angle and the saturated hydraulic conductivity in the depth direction through a one-dimensional random field modeling.

The parameters are assumed to be log-normally distributed (Gelhar 1986). We model the natural logarithms of the parameters as homogeneous Gaussian random fields with the following exponential autocorrelation coefficient function:

$\rho(\tau)=e^{-\frac{2 \tau}{r}}$

where $r$ is the correlation length; $\tau$ is the separation between two locations in the vertical direction. The correlation length is a measure of the spatial variability of the random field. A correlation length that is much larger than the depth of the soil slice implies a uniform soil profile; in this case, the soil parameters can be modeled by random variables. In the extreme case where the correlation length is close to zero, the values of the soil parameters at each location become independent from each other.

The random fields are discretized by the midpoint method (Der Kiureghian \& Ke 1988). The unit slice is divided into a number of equal layers; the randomized properties are assumed to be constant within each layer, represented by their values at the midpoints of the layers. Since homogenous fields are assumed, the mean $\mu$ and standard deviation $\sigma$ of the random variables corresponding to the discrete layers are constant over the entire field. The entries of the correlation matrix of the logarithms of the soil properties at each layer are evaluated with Equation (2) where $\tau$ is the distance between each pair of midpoints. Simulation of the soil parameters is performed by simulating the joint normal random variables of all layers and taking the exponential of the resulting samples. It is noted that the crosscorrelation coefficient between the two parameters is set to zero in this study.

\subsection{Infiltration analysis}

We utilize the Green and Ampt assumptions to simulate the infiltration process (Green \& Ampt 1911). That is, we assume that vertical infiltration causes a well-defined wetting front (see Figure 2). Above the wetting front, the soil is fully saturated while below the wetting front it continues to have its initial moisture content.

The soil column is divided into a number of equal-thickness layers with varying saturated hydraulic conductivities as discussed in Section 2.2. We approach the problem by assuming that the wetting front development takes place in a step-wise manner, whereby the wetting front advances by one layer in each computational step. That is, in step $j$ the wetting front is located at the bottom of the $j$ th layer. For the sake of simplicity, the initial moisture content $\theta_{0}$, soil porosity $\eta$ and suction head at wetting front $S$ are considered to be constants within the unit slice.

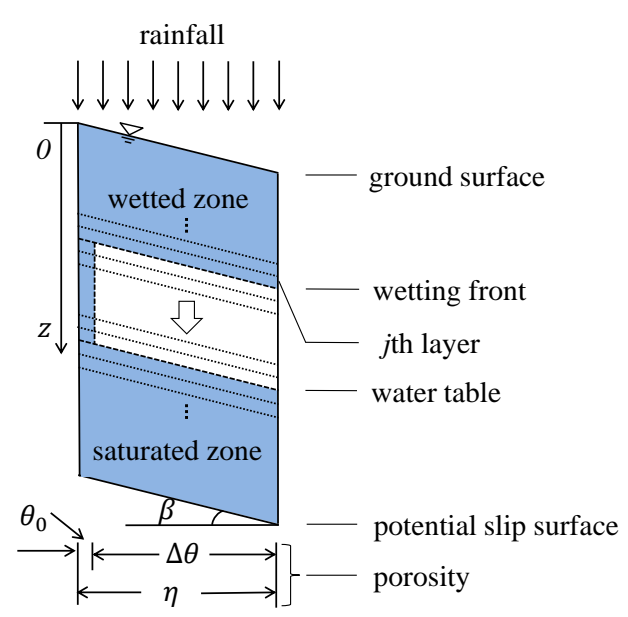

Figure 2. Green and Ampt model on the unit slice

This study focuses on short and intense rainfall events, during which the wetting front might not reach the water table. The infiltration process can be divided into two distinct phases (see Figure 3): vertical infiltration during the rainfall period and plug flow driven by the saturated hydraulic conductivity of the soil until formation of the new ground water table.

During the rainfall period [Phase I, see Figure $3(\mathrm{a})]$, the hydraulic gradient $i$ from the ground surface to the wetting front at layer $j$ along the vertical direction is obtained by application of Darcy's law: 


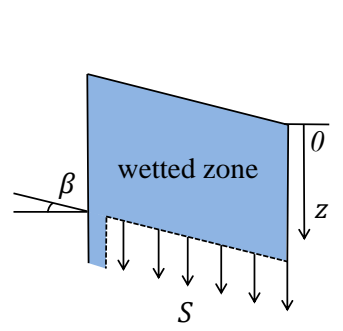

(a) Phase I: infiltration

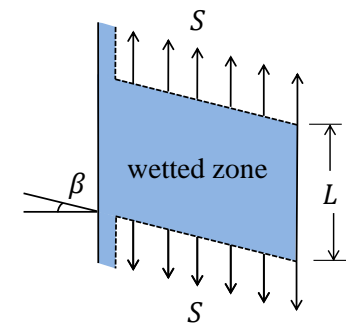

(b) Phase II: plug flow
Figure 3. Infiltration process

$i=\frac{z+S}{z}$

where $z$ is the depth of the wetting front at the bottom of the layer $j$; $S$ is the suction head at the wetting front. Note that Equation (3) neglects the influence of the ponding water depth. We can then evaluate the infiltration rate $f_{\text {in }}$ as follows:

$f_{\text {in }}=K_{e} \cdot i$

where $K_{e}$ is the effective vertical saturated hydraulic conductivity, namely the harmonic mean of the vertical saturated hydraulic conductivities at the soil layers within the wetted zone (Freeze \& Cherry 1979):

$K_{e}=\frac{j}{\sum_{l=1}^{j} \frac{1}{K_{l}}}$

in which $K_{l}$ is the saturated hydraulic conductivity of each wetted computational layer.

We assume that the rainfall supply is larger than the infiltration capacity. Therefore the cumulative infiltration will be governed by the infiltration capacity and can be obtained by

$F=z \cdot \Delta \theta$

in which $\Delta \theta$ is the change in moisture content, given as follows:

$\Delta \theta=\eta-\theta_{0}$

where $\eta$ is the porosity of the soil and $\theta_{0}$ is the initial moisture content (see Figure 2). Noting that $f_{\text {in }}=d F / d t$, we can obtain the cumulative infiltration time as follows:

$\int_{0}^{t} d t=\int_{0}^{z} \frac{\Delta \theta}{f_{\text {in }}} d z$

The second phase that follows the rainfall event is governed by plug flow. It is assumed that the suction at the bottom of the wetted zone equals the one at the top [see Figure 3(b)]. Therefore, the hydraulic gradient equals unity and the plug flow rate $f_{p}$ equals the harmonic mean of the saturated hydraulic conductivities of the layers corresponding to the wetted zone:
$f_{p}=K_{e}$

Therefore, the time needed for the wetting front to reach the bottom of each layer is obtained as follows:

$t= \begin{cases}\int_{0}^{z} \frac{\Delta \theta}{f_{i n}} d z \quad, t \leq T \\ T+\int_{z-L}^{z} \frac{\Delta \theta}{f_{p}} d z, t>T\end{cases}$

wherein $T$ is the duration of the rainfall event; $L$ is the length of wetting front exactly after rainfall stops [see Figure 3(b)]. Equation (10) can be evaluated numerically over the computational layers as

$t=\Delta z \cdot \Delta \theta \cdot \sum \frac{1}{f_{j}}$

where $\Delta z$ is the thickness of each layer; $f_{j}$ is the flow rate at the $j$ th computational step of either the infiltration or the plug flow phase depending on whether the time $t$ is smaller or greater than the duration of the rainfall event $T$. Through Equation (11) we obtain a mapping between the cumulative time and the wetting front development.

\subsection{Pore water pressure distribution}

The pore water pressure varies with time and space within one unit slice. In this study, emphasis is on the pore water pressure within the wetted zone and below the water table.

Within the wetted zone, the spatial variability of the saturated hydraulic conductivity may introduce large hydraulic gradients that will result in variable pore water pressure. Employing the plug flow assumption given by the Green and Ampt infiltration model, we assume that the effective flow rate of the wetted zone $f$ computed by Equations (4) and (9) equals the flow rate at each wetted layer (Liu et. al. 2008), i.e.

$f_{1}=f_{2}=\cdots=f_{l}=\cdots=f$

where $f_{l}$ is the flow rate at wetted layer $l$, computed by

$f_{l}=K_{l} \cdot i_{l}=K_{l} \cdot \frac{\Delta h_{l}}{\Delta z}$

wherein $i_{l}$ is the hydraulic gradient of layer $l ; \Delta h_{l}$ the change of hydraulic head within the $l$ th layer. Combining Equations (12) and (13), we obtain:

$\Delta h_{l}=\frac{f}{K_{l}} \cdot \Delta z$

At the bottom of $l$ th wetted layer, the hydraulic head $h_{l}$ is obtained by summing the incremental heads $\Delta h_{k}$ of each computational layer $k \leq l$

$h_{l}=h_{0}-\sum_{k \leq l} \Delta h_{k}$ 
in which $h_{0}$ is the initial hydraulic head at the top of the wetted zone, i.e. $h_{0}=0$ during Phase I [Figure 3(a)] and $h_{0}=-z_{0}-S$ during Phase II [Figure 3(b)], where $z_{0}$ is the initial elevation head. Since the hydraulic head $h_{l}$ consists of the pressure head $\psi_{l}$ and the elevation $z_{l}$, the pressure head at the bottom of $l$ th layer is evaluated as:

$\psi_{l}=h_{l}-z_{l}$

At the wetting front, the pressure head equals the suction head, i.e. $\psi_{j}=-S$. The pore water pressure can be computed as $u_{l}=\gamma_{w} \cdot \psi_{l}$, where $\gamma_{w}$ is the unit weight of water.

Below the water table, the soil is subjected to hydrostatic pressure $u=\gamma_{w} z^{*}=\gamma_{w} z_{w} \cos ^{2} \beta$, where $z^{*}$ is the projection of the equipotential line in vertical direction; $z_{w}$ is the depth of the soil layer under the ground water table (see Figure 4). The hydrostatic water pressure builds up when the ground water elevation rises as the wetting front reaches the water table.

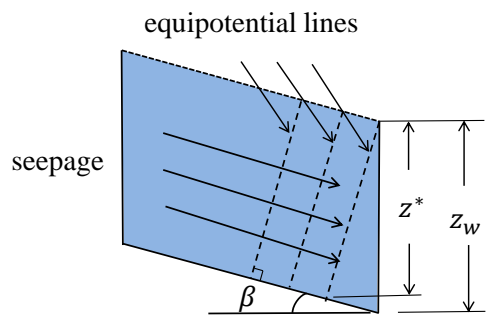

Figure 4. Hydrostatic pressure

\subsection{Reliability analysis}

Slope failure occurs when the factor of safety $F S$ is less than unity. Hence, the probability of failure of the slope is defined as

$P_{f}=\operatorname{Pr}[F S<1]$

In this study, we estimate $P_{f}$ by Monte Carlo simulation. Each realization of the random fields results in different values of the strength parameters and the saturated hydraulic conductivity at each soil layer. Based on the latter parameter value, we compute the pore water pressure distribution for each time step and each layer, as discussed in Section 2.4. We then substitute the strength parameters and pore water pressure into Equation (1) to obtain the factor of safety. Note that for each time step we evaluate the factor of safety for each potential slip surface, corresponding to the bottom of each layer. The overall factor of safety corresponds to the minimum value among all slip surfaces. The probability of failure for each time step is computed as the number of samples $N_{f}$ for which $F S$ is less than one divided by the total number of samples $N$ :

$P_{f} \approx \frac{N_{f}}{N}$

\section{EXAMPLE}

We are interested in the slope stability for short and intense rainfall events. We consider a slope with a $5 \mathrm{~m}$ deep layer of sandy soil with zero cohesion $c^{\prime}$, subjected to a $24 \mathrm{~h}$ intense rainfall. The slope angle is taken as $\beta=18^{\circ}$. The tangent of the friction angle $\tan \varphi^{\prime}$ and the saturated vertical hydraulic conductivity $K$ are modeled as lognormal random fields (Table 1). The correlation length for the two parameters is identical and we assume a zero crosscorrelation. The random fields are discretized into 100 layers. The water table is at a depth of $1.5 \mathrm{~m}$ below the ground surface. The parameter of the Green and Ampt model for sandy soil are taken from Rawls (1982) as given in Table 2. The number of Monte Carlo samples is set to $10^{4}$, which was found to give acceptable coefficient of variations of the probability estimates for the cases considered.

Table 1. The values and distributions of the input variables

\begin{tabular}{llll}
\hline Variable & Distribution & Mean $\mu$ & $\mathrm{CV}^{*}$ \\
\hline $\tan \varphi^{\prime}$ & Lognormal & 0.7002 & 0.1 \\
$K[\mathrm{~m} / \mathrm{h}]$ & Lognormal & $3.6 \times 10^{-3}$ & 2 \\
\hline${ }^{*} \mathrm{CV}$ stands for coefficient of variation $(=\sigma / \mu)$
\end{tabular}

Table 2. Parameters for Green and Ampt model

\begin{tabular}{llll}
\hline Variable & $\eta$ & $\theta_{0}$ & $S[\mathrm{~m}]$ \\
\hline Value & 0.437 & 0.102 & 0.1734 \\
\hline
\end{tabular}

\subsection{Influence of the correlation length}

The analysis is performed for two values of the correlation length $r$, that is $2 \mathrm{~m}$ and $0.5 \mathrm{~m}$. Figure 5 presents the development of the mean depth of the wetting front with time. The time period of interest is $240 \mathrm{hr}$ ( 10 days) after the start of the rainfall event.

Figure 5 shows that in the case of a smaller correlation length, the water flow will move slower towards the water table. This can be explained by the fact that a small correlation length implies a large variability within the wetted zone. The flow within the wetted zone is driven by the lower values of $K$, as evident from the use of the harmonic mean of the saturated hydraulic conductivity in Eq. (5); the higher the variability, the larger the likelihood of occurrence of low values. 


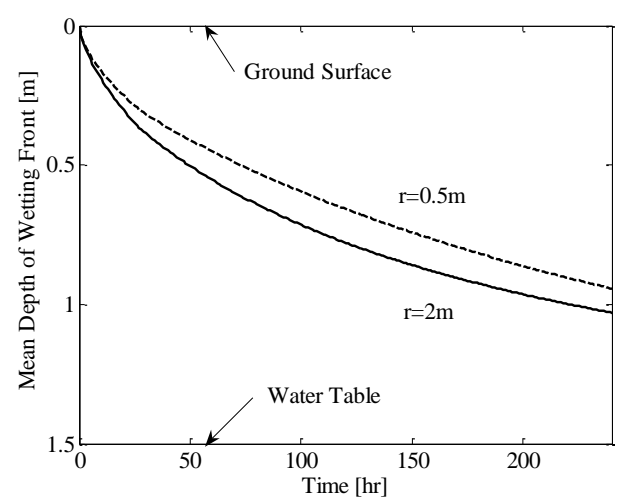

Figure 5. Development of the mean wetting front depth

Figure 6 shows the mean factor of safety of the slope with time. As also discussed in (Griffiths et al. 2011), the initial mean factor of safety is smaller for a smaller correlation length. A large correlation length implies a uniform soil profile and thus the most critical potential slip surface is at the base of the slope. On the other hand, a small correlation length implies vertically variable parameters and weak layers are likely to be present in most Monte Carlo realizations. The most-critical potential slip surface will likely occur in a weak layer, which will be present at a different location in each realization.

Since the pore water pressure increases due to the wetting front development, the mean factor of safety then decreases during the infiltration procedure for both cases. A faster decrease is observed for the case of the larger correlation length, since the water flow moves faster towards the water table, which causes a rise of the hydrostatic pressure.

During the rainfall event period, the mean factor of safety decreases rapidly and it reaches a transient lower value when rainfall stops. The reduction is higher for the case with a small correlation length, which implies that the likelihood of shallow slope failure is higher. A small correlation length introduces changes between large and small values of the hydraulic conductivity occurring at short distances within the wetted zone. Higher hydraulic gradients occur across the layers with low hydraulic conductivity values causing higher pressure to be built up immediately above such layers within the wetted zone. Therefore, a smaller correlation length will increase the likelihood of slip surfaces at intermediate layers above the wetting front.

After the rainfall event, the water flow transforms to plug flow [Phase II in Fig. 3(b)] and the mean factor of safety increases, since the likelihood of shallow slope failure decreases. Slope failure is now driven by the formation of the new ground water elevation that takes place when the wetting front reaches the water table. This happens faster in the case of a larger correlation length and hence the decrease of the mean factor of safety is larger in this case. A small correlation length implies that layers with low hydraulic conductivity exist in most realizations and these layers imped downward movement of infiltration water.

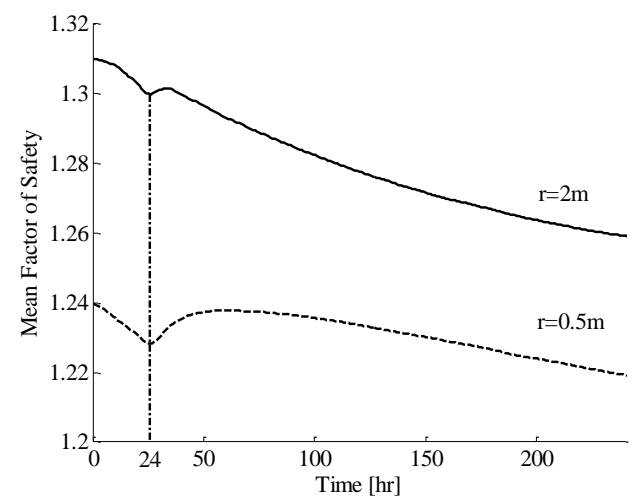

Figure 6. Influence of the correlation length on the factor of safety

Figure 7 depicts the transient evolution of the point-in-time probability of failure for the two considered correlation lengths. The initial probability of failure is higher when the correlation length is smaller, which corresponds to a smaller mean factor of safety. For this case, the probability of failure increases during the rainfall event, only to decrease again until the wetting front approaches the ground water table where the probability rises once more. The case corresponding to a larger correlation length presents a different behavior: During the rainfall event, shallow slope failure will mostly occur at the wetting front as there is no localized layer with lower relative hydraulic conductivity value that impedes infiltration and leads to porepressure building up within the wetting front. The wetting front will move faster towards the water table and hence the likelihood that the water table is reached within one realization is higher. This results in a smooth increase of the probability of failure in terms of time since the failure will be governed by the rise of the hydrostatic pressure.

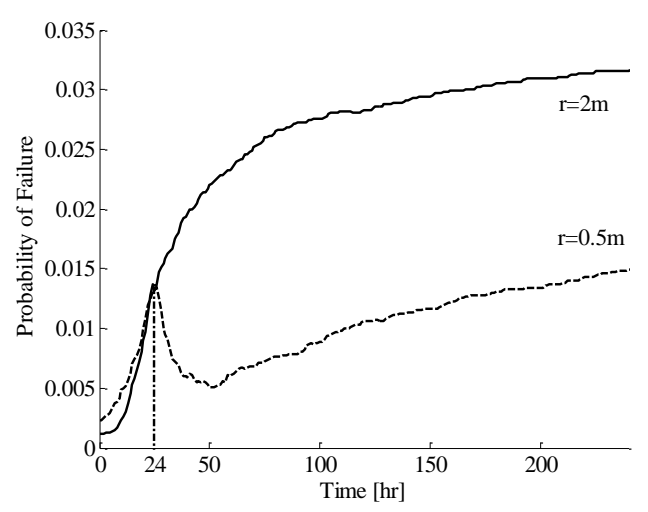

Figure 7. Influence of the correlation length on the probability of failure

\subsection{Influence of the coefficient of variation}

We now look at the influence of the coefficient of 
variation of the hydraulic conductivity on the stability and probability of slope for the case of a small correlation length $(0.5 \mathrm{~m})$; the coefficient of variation is varied from 1 to 3 . Figures 8 and 9 show the change of the mean factor of safety and the probability of failure with time.

Increasing the coefficient of variation will increase the variability within the wetted zone, which will cause the wetting front to move slower towards the water table and therefore failure will be governed by shallow slip surfaces. Moreover, a large coefficient of variation leads to steeper changes between small and large values of the hydraulic conductivity within the wetted zone; hence the pore water pressure at layers above the wetted zone increases, which leads to a larger probability of shallow slope failure and smaller corresponding mean factors of safety.

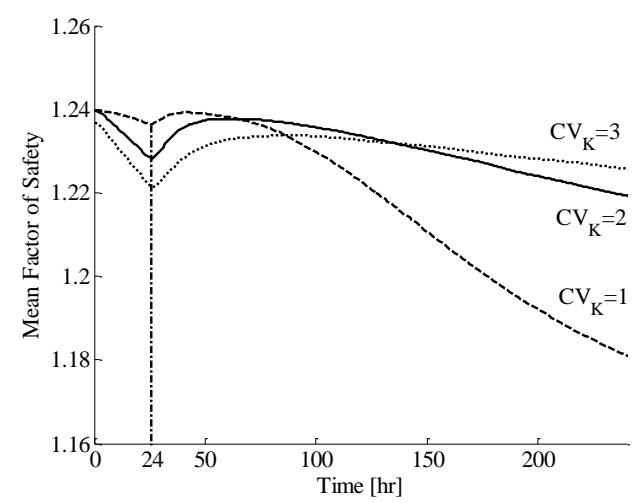

Figure 8. Influence of the coefficient of variation of the saturated hydraulic conductivity on the factor of safety for $r=0.5 \mathrm{~m}$

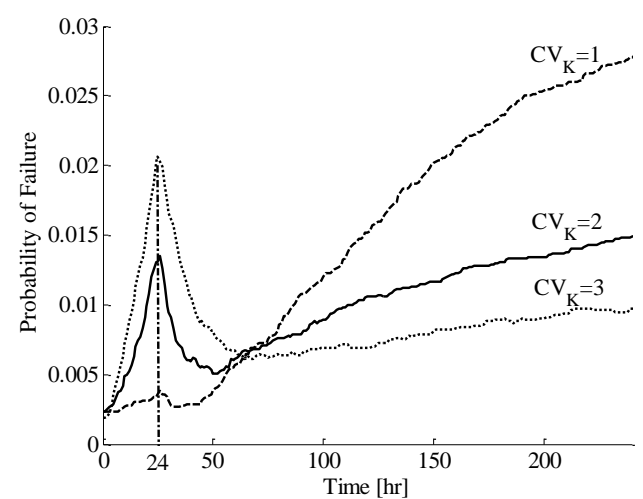

Figure 9. Influence of the coefficient of variation of the saturated hydraulic conductivity on the probability of failure for $r=0.5 \mathrm{~m}$

\section{CONCLUSION}

In this paper, we developed a simplified timedependent model to study the reliability of slopes subjected short-term and intense rainfall events. The model incorporates infinite slope stability analysis, one-dimensional infiltration analysis based on the Green and Ampt assumptions as well as a random field modeling of the effective friction angle and the saturated hydraulic conductivity of the soil. An example demonstrated the influence of the correlation length and coefficient of variation of the hydraulic conductivity on the development of the factor of safety and probability of failure with time. It is shown that a small correlation length and high coefficient of variation will favor shallow slope failure due to the decrease of the infiltration rate and the development of large pressure gradients within the wetted zone. In the cases with large correlation lengths and small coefficient of variation, the reliability is dominated by the rise of the hydrostatic pressure due to the fast wetting front development.

\section{REFERENCES}

Chen L. \& Young M.H. 2006. Green-Ampt infiltration model for sloping surface. Water resources research 42(7): W07420.

Corominas, J., Moya, J. \& Hürlimann, M. 2002. Landslide rainfall triggers in the spanish eastern pyrenees. Proc. 4th EGS Plinius Conference. Mallorca, Spain.

Der Kiureghian, A. \& Ke, J.-B. 1988. The stochastic finite element method in structural reliability. Probabilistic Engineering Mechanics 3(2): 83-91.

Freeze, A.R. \& Cherry, J. A. 1979. Groundwater. Upper Saddle River, NJ: Prentice Hall.

Gelhar L.W. 1986. Stochastic subsurface hydrology from theory to application, Water Resources Research 22(9): 135145.

Green, W. H. \& Ampt, G. A. 1911. Studies on soil physics, part I - the flow air and water through soils. Journal of Agricultural Science 4(1): 1-24.

Griffiths, D. V., Huang, J. \& Fenton, G. A. 2011. Probabilistic infinite slope analysis. Computers and Geotechnics 38(4): 577-584.

Liu J. T. , Zhang J. B. \& Feng J. 2008. Green-Ampt Model for Layered Soils with Nonuniform Initial Water Content Under Unsteady Infiltration. Soil Science Society of America Journal 72(4): 1041-1047.

Muntohar, A. S. \& Liao H. 2010. Rainfall infiltration: infinite slope model for landslides triggering by rainstorm. Natural Hazards 54(3): 967-984.

Phoon, K. K. \& Kulhawy, F. H. 1999. Characterization of geotechnical variability. Canadian Geotechnical Journal 36 (4): 612-624.

Polemio, M. 1997. Rainfall and Senerchia landslides, southern Italy. Proc. 2nd COBRAE, Rio de Janeiro.

Rawls, W. J., Brakensiek, D. L. \& Saxton, K. E. 1982. Estimation of soil water properties. Transactions of the ASAE 25(5): 1316-1320\&1328.

Santoso, A. M., Phoon, K., \& Quek, S. 2011. Effect of 1D Infiltration Assumption on Stability of Spatially Variable Slope. Geo-Risk 2011: Risk Assessment and Management. 704-711.

Wu, T. H. \& Abdel-Latif, M. A. 2000. Prediction and mapping of landslide hazard. Canadian Geotechnical Journal 37(4): 579-90.

Zêzere, J. L. Trigo, R. M. \& Fragoso, M., Oliveira, S. C. \& Garcia, R. A. C. 2008. Rainfall-triggered landslides in the Lisbon region over 2006 relationships with the north Atlantic Oscillation. Natural Hazards and Earth System Science 8(3): 483-499. 